\title{
Special issue on "recent advances in meiotic chromosome structure, recombination and segregation"
}

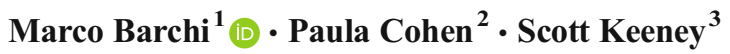

Received: 14 March 2016 / Accepted: 15 March 2016 / Published online: 29 March 2016

(C) Springer-Verlag Berlin Heidelberg 2016

\section{Preface}

Meiosis is a central process in eukaryotic life, in that it is critical to genetic reassortment and generation of gametes containing the correct haploid chromosome complement. As such, its proper execution is critical for the maintenance, evolution and health of the species, and it is thus highly regulated. Although the molecular genetics of fungal systems has provided key information for the understanding of basic mechanisms that govern chromosome segregation during meiosis, the study of meiosis in multicellular organisms is more difficult. Indeed, the need for proper communication among germ cells and with somatic cells within gonads further increases the complexity of mechanisms that control meiotic chromosome dynamics. For these reasons, the basic mechanisms and regulation of meiosis in higher eukaryotes are still far from fully understood and represent a challenge for present and future generations of researchers. In this Special Issue (SI), by looking at both unicellular and multicellular organisms as models, the authors provide an updated view of key processes and molecular players that regulate meiotic events. Such players are required for the establishment of the unique properties of meiotic chromosome that underlie the formation and

Marco Barchi

marco.barchi@uniroma2.it

1 Department of Biomedicine and Prevention, Section of Anatomy, University of Rome Tor Vergata, Rome 00133, Italy

2 Center for Reproductive Genomics, Department of Biomedical Sciences, Cornell University, Ithaca, NY 14853, USA

3 Howard Hughes Medical Institute, Molecular Biology Program, Memorial Sloan-Kettering Cancer Center, New York, NY 10065, USA placement of crossovers and that promote and monitor proper partition of meiotic chromosomes among daughter cells. The Issue has been entitled "Recent Advances in Meiotic Chromosome Structure, Recombination and Segregation", and it consists of fourteen exciting manuscripts (seven original research articles and seven reviews) that present the most recent discoveries and views in the field.

\section{Original research articles}

During prophase of the first meiotic division, double strand breaks (DSBs) are produced by SPO11 protein, and repair of these breaks in mammals promotes recombination, pairing and synapsis between the homologous chromosomes (homologs). Using an elegant mouse model carrying rearranged sex chromosomes, Decarpentrie et al. (2015) provide new insight into patterns of recombination between the $\mathrm{X}$ and $\mathrm{Y}$ chromosomes in male mice.

In addition to DSB formation per se, timing and level of DSBs are also thought to be crucial to guarantee accurate progression of recombination. Using a Spo11-transgenic mouse model that expresses reduced levels of SPO11, Faieta et al. (2015) determine the level of DSBs needed to support proper pairing and synapsis of homologs. They show that, during spermatogenesis, delayed chromosome synapsis between homologs that received "too few" DSBs at leptonema, can be rescued by a late-forming wave of DSBs.

DSB formation and recombination are tightly integrated with higher-order chromosome structure. Pairs of sister chromatids are organized into a series of loops anchored at their bases along a structural axis called the axial element. At the pachytene stage, homologs are held together along their lengths by a tripartite structure called the synaptonemal complex, which comprises two lateral elements (formerly the axial element of 
each homolog) held together by transverse filaments. Axial elements are enriched with several protein components, which in fission yeast Schizosaccharomyces pombe includes Rec8 and Pds5. Using super-resolution structured illumination microscopy, Ding et al.(2015) show that in addition to their established role in meiotic prophase chromosome compaction, these cohesins are also required for the spatial alignment of homologous chromosomes. Moreover, the cohesins form a physical platform for the recognition of, and pairing and recombination between, homologous chromosomes.

In mammalian meiosis, spermatocytes with incomplete synapsis are often eliminated by apoptosis. A key surveillance mechanism operating in these cells is Meiotic Silencing of Unsynapsed Chromosomes (MSUC), which silences essential genes and promotes elimination of synapsis-defective cells. Although this mechanism has been shown to function efficiently during mammalian spermatogenesis, particularly at the level of the $\mathrm{X}$ and $\mathrm{Y}$ chromosomes, whether it also operates in female mammalian meiosis is unclear. Cloutier et al. (2015) demonstrate that MSUC also occur in oocytes. However, the silencing mechanism is less efficient than in males, and it is influenced by gonadal sex.

$\mathrm{CO}$ formation between $\mathrm{X}-\mathrm{Y}$ chromosomes in mammals is particularly challenging, as it hinges on efficient recombination within a very small region of homology, the pseudoautosomal region (PAR). In a mouse model expressing a single splicing isoforms of SPO11 (Spo11 $\beta$-only mouse), CO formation within the PAR often fails, and spermatocytes are eliminated by apoptosis following the activation of a prophase-I spindle-checkpoint. Using this mouse model, Faisal and Kauppi (2015) show that in mammals, X-Y recombination frequency might change depending on the mouse strain genetic background, and set the threshold of $\mathrm{X}-\mathrm{Y}$ crossover failure compatible with male fertility.

In most sexually reproducing organisms, the key products of the recombination process are crossovers (COs), which physically connect homologs so they orient and segregate properly on the first meiotic spindle. To ensure that each chromosome receives at least the one $\mathrm{CO}$ event that ensures proper segregation, $\mathrm{CO}$ frequency and placement are stringently regulated. Over the years, genetic identification of CO-defective mutants, and cloning of the genes involved has allowed the identification of many proteins involved in this process. In this SI, using a gene trap strategy, Sun et al. (2015) identify Fancj as a critical factor required for both spermatogonial proliferation and establishment of the appropriate $\mathrm{CO}$ number in mouse spermatocytes. This study further expands the catalog of genes necessary to proper $\mathrm{CO}$ formation in mammals.

The meiosis-specific events described above all occur within the nucleus, in the context of a very rapid and dynamic movement of the chromosomes. Telomere-led prophase movements of chromosomes have been proposed to move chromosomes relative to one another, helping establish homologous interactions during pairing, resolve chromosome entanglements, and also regulating $\mathrm{CO}$ placement. Key for such processes is the maintenance of telomere integrity. By studying cyclin $E 1$ and $E 2$ knockout mice, Manterola et al. (2015) show that E-type cyclins are master modulators of such processes promoting telomere integrity and correct synapsis of the homologs.

\section{Reviews}

An important aspect of this SI is that experimental data are contextualized within broad and in-depth reviews illustrating main findings of the field in recent years. The review from Ribeiro et al. (2015) focuses on DNA metabolism during meiosis, illustrating how different homologs of the single-stranded DNA (ssDNA) binding protein RPA might provide meiotic identity to ssDNA intermediates and be essential to meiotic recombination and progression. Fabig et al. (2015) focus on the mechanisms of segregation of univalent sex chromosomes (i.e. chromosomes that have no pairing partner or that have a partner but do not establish any direct physical connection with it), describing how different species overcome the challenge of segregating these chromosomes. The review by Bomblies et al. (2016) covers a different chromosome segregation challenge, namely how chromosome segregation problems are resolved when polyploidy first arises in autopolyploid eukaryotic organisms. By comparing newly formed (neo)-autotetraploids and fully evolved autotetraploid species, they suggest that evolution of stable autopolyploid organisms is made possible by an increase in distance between COs, a phenomena known as increased interference distance. Termolino et al. (2016) highlight that $\mathrm{CO}$ formation and positioning along chromosomes are strictly linked to high order chromatin configuration in that specific chromatin epigenetic modifications might be consistently found in recombination-free and permissive regions. Thus, collecting direct and indirect data from different organisms, they summarize recent advances in the field and describe regions and chromatin marks involved in this process.

In diploid cells at metaphase-I, COs between homologous (non-sister) chromatids, along with sister chromatid cohesion, are essential for the regular segregation of the chromosomes to opposite poles of the spindle. Connectedness between the homologs allows the centromere/kinetochore complex to be placed under mechanical tension, promoting both proper orientation of the homologs and maintaining inoperative the "spindle assembly checkpoint" (SAC), with consequent progression to anaphase. The review of Kakui and Sato (2015) describes studies mainly made in yeast, and underlines how meiosis-specific regulation of kinetochore-microtubule attachments have differentiated from mitosis to meiosis in order to ensure faithful and efficient chromosome segregation after meiotic recombination. On the same subject, Touati and Wassmann (2015) extensively reviews SAC mechanisms in mammalian female meiosis, 
further summarizing recent advances in the field. Telomere metabolism is the focus of the review of Reig-Viader and coauthors (2015). Here, in addition to the role of E-cyclins described earlier, the reader will find insight regarding how telomere homeostatic maintenance is connected with mammalian fertility, and how detection of telomere components might potentially serve as a tool to develop biomarkers for fertility diagnoses, linking basic research to applied biology.

It is our hope that this collection of manuscripts and reviews will be of use to the meiosis research community, especially to students and young researchers who recently entered the field. Despite recent advances, further efforts are needed to uncover the molecular mechanisms that control chromosome dynamics and recombination in larger eukaryotes, such as mouse and humans. The next several years of research should prove highly fruitful as new genetic models and technologies continue to provide new instruments for investigation.

Marco Barchi, Ph.D.

Guest Editor

Paula Cohen, Ph.D.

Associate Editor

Scott Keeney, Ph.D.

Associate Editor

\section{References}

Bomblies K, Jones G, Franklin C, Zickler D, Kleckner N (2016) The challenge of evolving stable polyploidy: could an increase in "crossover interference distance" play a central role? Chromosoma. doi: 10.1007/s00412-015-0571-4

Cloutier JM, Mahadevaiah SK, ElInati E, Tóth A, Turner J (2015) Mammalian meiotic silencing exhibits sexually dimorphic features. Chromosoma. doi:10.1007/s00412-015-0568-Z

Decarpentrie F, Ojarikre OA, Mitchell MJ, Burgoyne PS (2015) Recombination between the mouse $\mathrm{Y}$ chromosome short arm and an additional Y short arm-derived chromosomal segment attached distal to the X chromosome PAR. Chromosoma. doi:10.1007/ s00412-015-0559-0

Ding DQ, Matsuda A, Okamasa K, Nagahama Y, Haraguchi T, Hiraoka Y (2015) Meiotic cohesin-based chromosome structure is essential for homologous chromosome pairing in Schizosaccharomyces pombe. Chromosoma. doi:10.1007/s00412-015-0551-8

Fabig G, Müller-Reichert T, Paliulis LV (2015) Back to the roots: segregation of univalent sex chromosomes in meiosis. Chromosoma. doi: 10.1007/s00412-015-0550-9

Faieta M, Di Cecca S, de Rooij DG, Luchetti A, Murdocca M, Di Giacomo M, Di Siena S, Pellegrini M, Rossi P, Barchi M (2015) A surge of late-occurring meiotic double-strand breaks rescues synapsis abnormalities in spermatocytes of mice with hypomorphic expression of SPO11. Chromosoma. doi:10.1007/s00412-0150544-7

Faisal I, Kauppi L (2015) Sex chromosome recombination failure, apoptosis, and fertility in male mice. Chromosoma. doi:10.1007/ s00412-015-0542-9

Kakui Y, Sato M (2015) Differentiating the roles of microtubuleassociated proteins at meiotic kinetochores during chromosome segregation. Chromosoma. doi:10.1007/s00412-015-0541-x

Manterola M, Sicinski P, Wolgemuth DJ (2015) E-type cyclins modulate telomere integrity in mammalian male meiosis. Chromosoma. doi: 10.1007/s00412-015-0564-3

Reig-Viader R, Garcia-Caldés M, Ruiz-Herrera A (2015) Telomere homeostasis in mammalian germ cells: a review. Chromosoma. doi:10. 1007/s00412-015-0555-4

Ribeiro J, Abby E, Livera G, Martini E (2015) RPA homologs and ssDNA processing during meiotic recombination. Chromosoma. doi:10.1007/s00412-015-0552-7

Sun X, Brieño-Enríquez MA, Cornelius A, Modzelewski AJ, Maley TT, Campbell-Peterson KM, Holloway JK, Cohen PE (2015) FancJ (Brip1) loss-of-function allele results in spermatogonial cell depletion during embryogenesis and altered processing of crossover sites during meiotic prophase I in mice. Chromosoma. doi:10.1007/ s00412-015-0549-2

Termolino P, Cremona G, Consiglio MF, Conicella C (2016) Insights into epigenetic landscape of recombination-free regions. Chromosoma. doi:10.1007/s00412-016-0574-9

Touati SA, Wassmann K (2015) How oocytes try to get it right: spindle checkpoint control in meiosis. Chromosoma. doi:10.1007/s00412015-0536-7 